\title{
EVALUACIÓN DE LA PRESENCIA INCAICA EN EL CENTRO OESTE ARGENTINO: LOS SITIOS DE LA QUEBRADA DE CONCONTA (SAN JUAN, ARGENTINA)
}

\section{EVALUATION OF THE INCA PRESENCE IN CENTRAL WEST ARGENTINA: THE SITES OF CONCONTA RAVINE (SAN JUAN, ARGENTINA)}

\author{
Alejandro García ${ }^{1}$ \\ ${ }^{1}$ Cigeobio (UNSJ-CONICET), FCEFyN-UNSJ. Av. José Ignacio de la Roza Oeste 727, J5402DCH \\ San Juan, Argentina. Email: alegarcia@unsj.edu.ar
}

Presentado: 06/02/2020

Aceptado: $22 / 5 / 2020$

\section{Resumen}

Uno de los principales hallazgos arqueológicos recientes de San Juan fue el de un conjunto articulado de sitios identificados inicialmente como incaicos en una zona carente de evidencias de ese período. Se trata de una serie de estructuras y tramos de caminos localizados en la parte alta de la Quebrada de Conconta, en el noroeste de la provincia. La importancia del hallazgo motivó su inmediata inclusión en el listado de sitios del Proyecto Internacional Qhapaq Nan. Sin embargo, un análisis detallado de este registro permite brindar una interpretación completamente diferente. En este sentido, el presente artículo aborda la evaluación global de toda la información disponible sobre estos sitios, cuyos resultados muestran un origen y cronología distintos a los propuestos.

Palabras clave: inca, dominación incaica, Collasuyu, San Juan

\section{Abstract}

One of the main recent archaeological findings of San Juan was that of an articulated set of presumably Inca sites in an area devoid of evidence of that period. It is a series of structures and road sections located in the upper part of the Quebrada de Conconta, in the northwest of the province. The importance of the finding motivated its immediate inclusion in the list of sites of the International Project Qhapaq Nan. However, a detailed analysis of this record allows us to provide a completely different interpretation. In this sense, this article deals with the global evaluation of all the information available on these sites, whose results show a different origin and chronology than those proposed.

Key words: inca, inca domination, Collasuyu, San Juan 


\section{Introducción}

El registro arqueológico arquitectónico incaico es relativamente abundante en la vertiente oriental andina del extremo SE del Collasuyu, fundamentalmente en las zonas aledañas a la cordillera (Figura 1). El sector con mayor cantidad de sitios incaicos en la provincia de San Juan es la Reserva de San Guillermo, en el norte de San Juan (Gambier y Michieli 1986, 1992); el noroeste de Mendoza es el otro sector más destacado de la región (Bárcena 1979, 1988, 1998; Schobinger y Bárcena 1971; etc.), si bien su registro es cuantitativamente mucho menor. Entre ambos se extiende un espacio de más de $300 \mathrm{~km}$ en el que la materialidad incaica es muy diversa y se encuentra distribuida de manera irregular. Aunque se ha sugerido que dentro de este espacio el contacto entre ambas vertientes cordilleranas se realizaba a través de varias vías transversales (e.g. Bárcena 2001; Stehberg 1995) aún no se han hallado las evidencias correspondientes, por lo que se ha propuesto la posible ausencia de vías de comunicación incaicas de uso frecuente entre $31^{\circ}$ y $32^{\circ}$ (García 2017).

De ahí que resultara significativa la propuesta que involucraba la presencia articulada de cinco sitos incaicos y de varios tramos de vialidad imperial en una de esas posibles vías (la Quebrada de Conconta), tanto por la llamativa concentración de conjuntos arquitectónicos como por su relación con el cruce transcordillerano a través del Paso de Las Tórtolas (Michieli, 2011, 2015; Michieli et al. 2005, 2010). A pesar de las dudas planteadas sobre esta asignación cultural por el autor (García 2007a), quien conocía estos sitios desde el año 2000, la falta de publicación de una evaluación detallada de aquella propuesta contribuyó a su perduración y abierta aceptación. Así, tanto a nivel político y académico como en el ideario público sanjuanino aquellos sitios son considerados como parte de los mayores exponentes de la dominación incaica local, y su adscripción incaica ha sido aceptada por diversos arqueólogos (Bárcena 2006; Durán et al. 2014; Vitry 2007), organismos oficiales (e.g., el Consejo Federal de Inversiones y el Senado de la Nación) y periódicos locales. Por otro lado, estos sitios fueron incorporados inicialmente al listado correspondiente a la Declaratoria del Camino del Inca como Patrimonio Cultural de la Humanidad, aunque luego fueron sustituidos (UNESCO 2014).

A fin de contribuir a clarificar la situación acerca del carácter inca de estos sitios, este artículo presenta un análisis de toda la información disponible, una evaluación de la posible relación entre las construcciones localizadas en el sector proximal de la Quebrada de Conconta y el período incaico y una propuesta alternativa sobre su origen y cronología. 


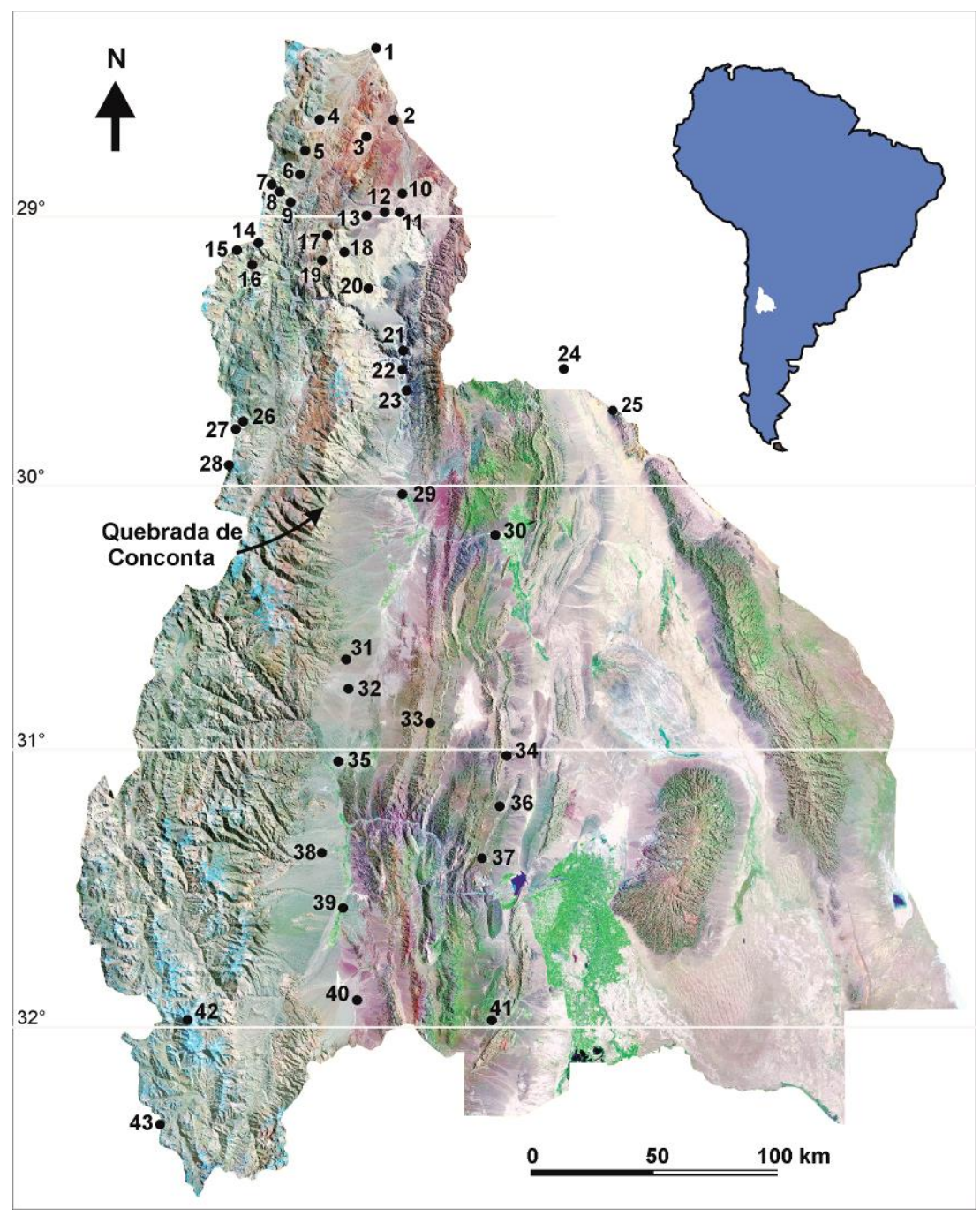

Figura 1. Sitios incaicos o con registro arqueológico incaico de San Juan y sectores limítrofes. Ubicación de los sitios incaicos o con evidencias incaicas de San Juan y zonas limítrofes, y del trazado de la vialidad estatal. 1) Río Blanco. 2) A Infiernillo. 3) Cajoncito Verde de la Brea. 4) Río del Macho Muerto 4. 5) Río de la Sal 2. 6) Río de la Sal 1. 7) Río Tambos Aguas Arriba. 8) Río Tambos Confluencia. 9) $\mathrm{A}^{\mathrm{o}}$ de los Tambillos. 10) Los Sapitos. 11) Santa Rosa. 12) Tambo del Indio. 13) Las Olorosas. 14) $C^{\circ}$ El Toro. 15) Paso Valeriano. 16) Sin nombre. 17) La Gloria. 18) Pircas Blancas. 19) Pircas Negras. 20) Huesos Quebrados. 21) Alcaparrosa. 22) Sin nombre. 23)

Pircas Negras. 24) Guandacol. 25) Paso del Lámar. 26) A La Deidad. 27) Río Frío. 28) C ${ }^{\circ}$

Tórtolas. 29) Angualasto. 30) Pachimoco. 31) Tocota. 32) Caminca 5. 33) La Invernada. 34)

Talacasto. 35) Tamberías. 36) Matagusanos. 37) La Dehesa. 38) Barrealito. 39) Barreal. 40) Tambería del Leoncito. 41) Pedernal. 42) $C^{\circ}$ Mercedario. 43) Tambería Paso de Valle Hermoso. Obsérvese el vacío notable en el sector cordillerano entre los sitios 28 y 42.

\section{Antecedentes regionales}

El comienzo de los estudios incaicos en San Juan se remonta a principios del siglo XX, con la excavación del sitio Barrealito (Debenedetti 1917). El siguiente trabajo sistemático fue realizado por Berberián en el Tambo de Tocota recién en la década de 1960 (Berberián et al. 1981). Gambier y Michieli (1986, 1992) desarrollaron 
relevamientos en el extremo $\mathrm{NO}$ y realizaron algunas reflexiones sobre las causas de la anexión de San Juan al Tawantinsuyu. Los trabajos posteriores se vinculan fundamentalmente con la prospección de nuevos sectores (e.g. Bárcena 2001, 2005; García 2007b), con la vialidad incaica (Michieli 2001, García 2011, 2017) y con la excavación de algunos sitios (Bárcena et al. 2008; Cahiza y Ots 2005; García 2015; Rodriguez y García 2016). Entre estos últimos trabajos se cuentan los estudios realizados en la Quebrada de Conconta (Michieli 2011, 2015; Michieli et al. 2005, 2010), aquí analizados.

\section{Los sitios analizados: ubicación y funcionalidad propuesta}

La Quebrada de Conconta está ubicada en la zona montañosa alta del NO de San Juan, a $30^{\circ} 01^{`} \mathrm{~S}$ y $69^{\circ} 35^{`} \mathrm{O}$, y conecta el piedemonte oriental cordillerano $(2900 \mathrm{~m}$ s.n.m.) con el Valle del Cura (3900 m s.n.m.), luego de atravesar alturas cercanas a los $5000 \mathrm{~m}$ (Figura 2). La quebrada tiene $19 \mathrm{~km}$ de extensión y dista unos $12 \mathrm{~km}$ del Valle del Cura, que a su vez se encuentra a aproximadamente $15 \mathrm{~km}$ del límite con Chile. En su parte superior el camino actual asciende una ladera que presenta un declive muy marcado, conocida como Cuesta de Vallejo.

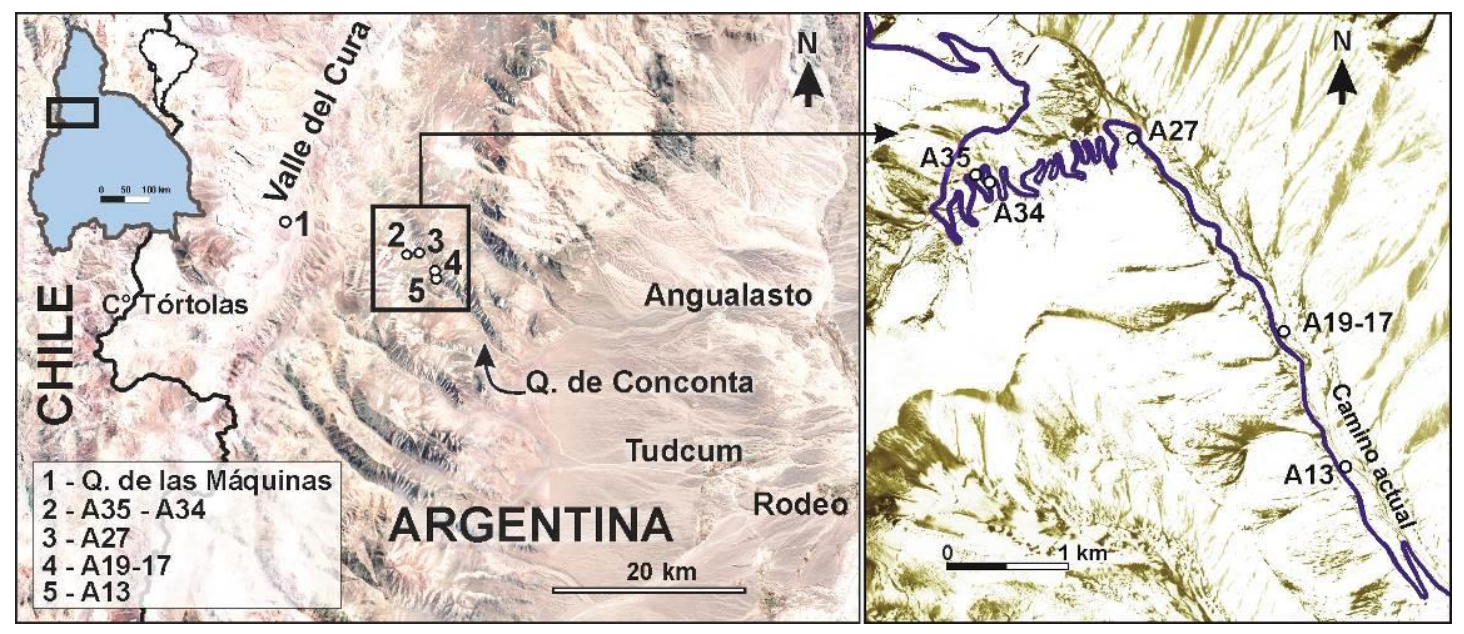

Figura 2. Ubicación de la Quebrada de Conconta y de los principales sitios mencionados.

Los sitios analizados se encuentran en este tramo superior de la quebrada, fundamentalmente al pie y en el medio de la cuesta. Se trata de cinco grupos de estructuras (Rincón de Vallejo -A35-, Rincón de Vallejo 2 -A34-, Cuesta de Vallejo -A27-, A19/A17 y A13) y tres tramos principales de camino (A19/17, A15/14, A11/5, y A100). Michieli et al. (2005) realizaron una detallada descripción de todas las estructuras, basada en su exhaustiva observación y en la excavación de las habitaciones 3, 13 y 15 del sitio Rincón de Vallejo. Estas autoras propusieron que estos sitios constituían "tambos de alojamiento" ubicados a 4540 m s.n.m, y una serie de construcciones intermedias vinculadas entre sí y con tramos de la vialidad imperial ubicados en el fondo de la Quebrada de Conconta y en las cercanías del portezuelo homónimo. Todo este conjunto había estado destinado a "formar un 
sistema de apoyo" para facilitar el paso de dicho portezuelo y poder acceder al Valle del Cura y desde allí a la vertiente occidental andina a través del Paso de las Tórtolas. La adscripción de los sitios de Conconta al período incaico se basó exclusivamente en una serie de rasgos arquitectónicos considerados como incas. Como refuerzo argumental se sostuvo que otro "rasgo común de las ruinas incaicas" es la "reutilización de las construcciones en épocas modernas". La ausencia de registro arqueológico asociado se explicaría por el hecho de que la construcción de estos sitios fue planificada (y en consecuencia, limpia y ordenada), y súbitamente habría sido interrumpida por la caída del poder central en el Cuzco a manos de los españoles (Michieli et al. 2005: 27).

\section{Análisis y discusión}

\section{Características arquitectónicas diagnósticas}

La filiación incaica de los sitios de la Quebrada de Conconta se basó en la presencia de "los rasgos arquitectónicos más característicos (...) presentes en casi todas las instalaciones incaicas". Éstos son: a) la construcción de grupos de habitaciones conectadas de planta rectangular combinadas con habitaciones de planta circular en menor número (generalmente utilizadas como depósitos); b) la selección de las piedras para la construcción; c) el cuidado en la cimentación; d) las esquinas de las construcciones rectangulares en ángulo recto o ligeramente redondeadas por el lado exterior; e) la unión de las piedras de los muros con mortero de barro; f) el muro con doble hilada con espesor constante; g) el remate trabado de las paredes (en vanos, aberturas, hornacinas y esquinas); h) el nacimiento de paredes como continuación o a partir de muros de contención; i) la forma trapezoidal de hornacinas o nichos, vanos y aberturas en general; j) los vanos cerrados con dintel de una sola pieza de piedra o los vanos abiertos hasta el techo sin dintel; k) el ancho constante de los vanos; l) la presencia de jambas de piedra en los vanos; 1l) la presencia de plataformas artificiales de tamaño diverso; m) la presencia de unidades constructivas menores dentro de las habitaciones como poyos, depósitos, recintos o cajas para la crianza de cuyes o para acciones ceremoniales; n) la presencia de miradores y torres (Michieli et al. 2005: 22).

Como puede observarse, se trata de un conjunto de componentes que no son exclusivos de un determinado estilo arquitectónico, sino que pueden hallarse en construcciones de distintos períodos y lugares. Así, elementos como los muros de doble hilada, el "símil sillar", las banquetas, los escalones aterrazados, el uso de mortero de tierra o barro, etc., se encuentran en construcciones hispánicas, algunas de ellas actualmente en uso (Figura 3). 


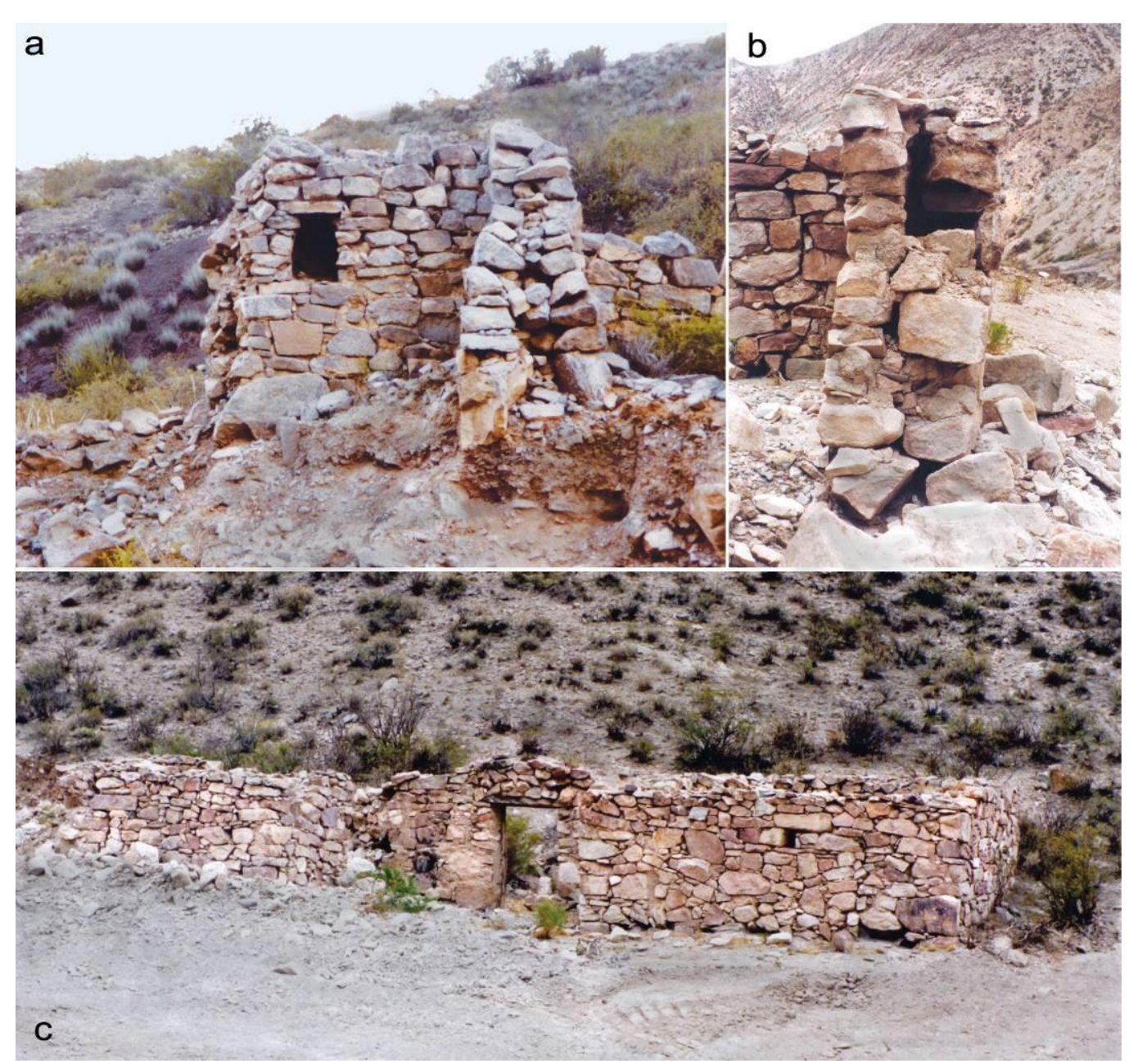

Figura 3. Construcciones modernas con "rasgos incaicos". a) Hornacina y muro de doble hilada de Los Blanquitos; b y c) Muro de doble hilada y vista general de una construcción de la zona del Acequión (sur de San Juan).

Por ejemplo, una construcción moderna localizada en el camino precordillerano que une la localidad de El Acequión con el valle de Calingasta, muestra muros de doble hilada, construcción en símil sillar, remate trabado de las paredes, hornacinas, banquetas, conjunto de habitaciones cuadrangulares con paredes unidas en ángulo recto, uso de mortero de tierra, etc. Algo similar ocurre con numerosas construcciones de uso reciente o actual, en las que no se observa una ocupación incaica previa, como el puesto El Toro (en la zona de Gualcamayo) o el puesto Los Corrales, en el Parque Nacional San Guillermo. Un caso interesante es el de Los Blanquitos, un sitio relacionado con la explotación de oro en la mina de Gualilán (Ullum, San Juan) durante el siglo XIX, que presenta varias de las características "indudablemente incaicas" consideradas en Conconta: muros de doble hilada, hornacinas, construcción en símil sillar, hastiales, excavación del suelo, etc. Sin embargo, la total ausencia de evidencias arqueológicas asociables al período incaico no permitió su vinculación con la dominación incaica regional (Bárcena 2005). 
La distribución de los "rasgos arquitectónicos incas" tampoco apoya la propuesta discutida: en lugar de actuar como un conjunto de elementos que se reitera "en casi todos" los sitios descriptos, estas características constructivas sólo aparecen dispersas y de manera infrecuente en los diferentes conjuntos (Tabla 1).

\begin{tabular}{|c|c|c|c|c|c|c|c|c|}
\hline "Rasgo arquitectónico" & $\mathbf{R V}$ & RV2 & $\mathrm{CV}$ & A19/17 & A15/14 & A13 & A11/15 & A100 \\
\hline \multicolumn{9}{|l|}{ Muro doble hilada } \\
\hline \multicolumn{9}{|l|}{ Símil sillar } \\
\hline \multicolumn{9}{|l|}{ Excavación del suelo } \\
\hline \multicolumn{9}{|l|}{ Plataformas } \\
\hline \multicolumn{9}{|l|}{ Escalones aterrazados } \\
\hline \multicolumn{9}{|l|}{ Uso de mortero de tierra } \\
\hline \multicolumn{9}{|l|}{ “Banqueta" } \\
\hline \multicolumn{9}{|l|}{ “Mirador" } \\
\hline \multicolumn{9}{|l|}{ Jamba } \\
\hline \multicolumn{9}{|l|}{ Vanos trapezoidales } \\
\hline \multicolumn{9}{|l|}{ Camino con "muro de } \\
\hline \multicolumn{9}{|l|}{ Camino delineado } \\
\hline Depósito para crianza de cuyes & & & & & & & & \\
\hline
\end{tabular}

Tabla 1. "Rasgos arquitectónicos" presentes en las estructuras de referencia (según Michieli et al. 2005).

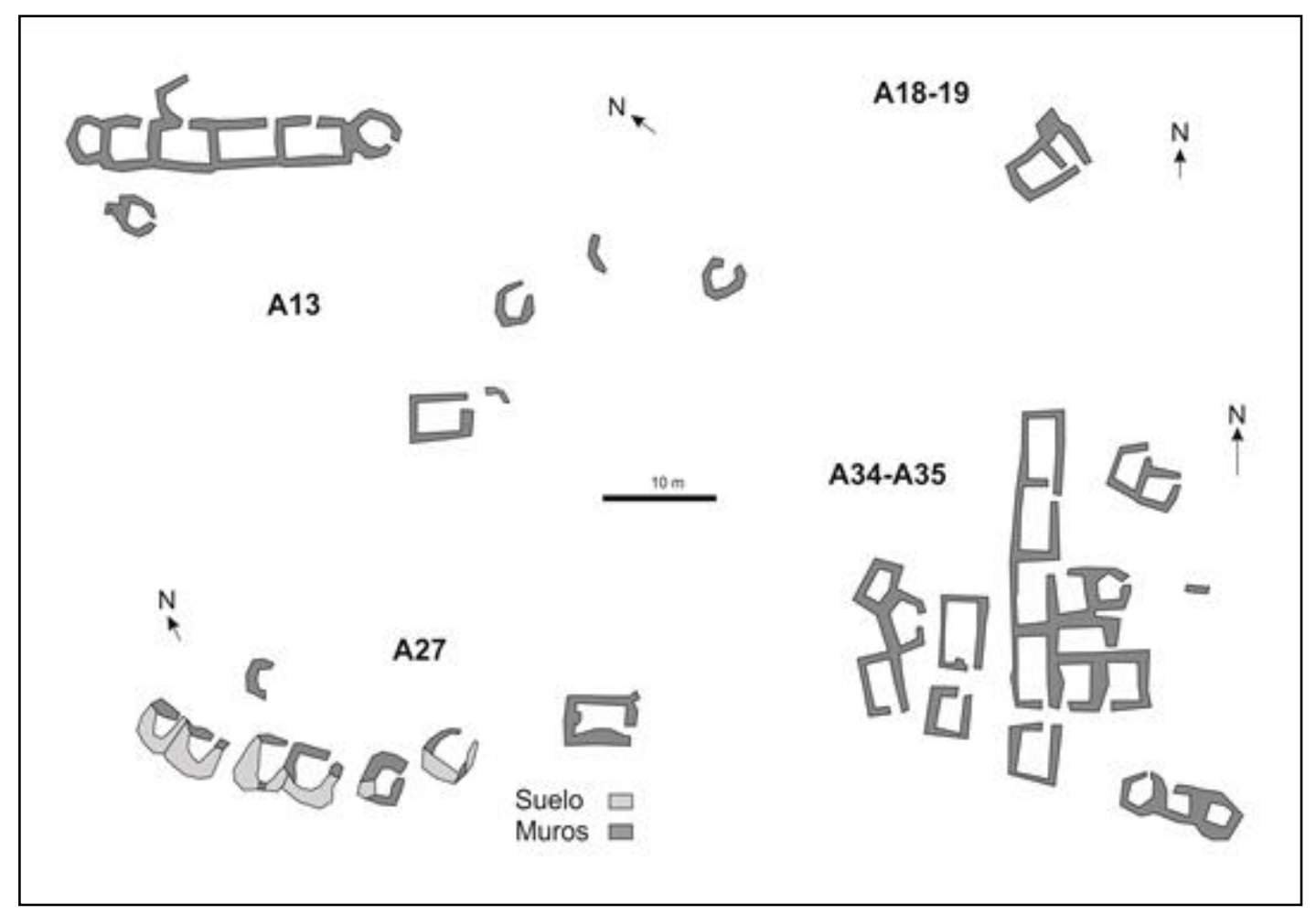

Figura 4. Forma de las estructuras de los sitios de Conconta (modif. de Michieli et al. 2005). 
Por otra parte, varios de aquellos rasgos arquitectónicos no han sido descriptos ni han podido ser observados en las construcciones. Entre ellos se destacan "los vanos cerrados con dintel de una sola pieza de piedra", las "hornacinas o nichos" trapezoidales y las torres.

Aunque no definitoria, la ausencia de diseños incaicos típicos en las construcciones (kallancas, RPCs, plazas intramuros, etc.) es otro elemento importante a considerar, ya que los sitios analizados presentan una diagramación muy diferente a la de los sitios incas del área. Por el contrario, se observan en Rincón de Vallejos, Rincón de Vallejos 2, Cuesta de Vallejos y A19-17 series de habitaciones dispuestas en patrones muy disímiles de los registrados en los tambos de la región (Figura 4).

\section{Determinación específica de los rasgos arquitectónicos}

Más allá de los problemas de considerar algunos rasgos arquitectónicos como elementos diagnósticos de la presencia incaica, subyacen dudas acerca de su propia identificación, presencia e interpretación funcional. Estos problemas se observan fundamentalmente en los casos del mirador y del depósito para criar cuyes. El mirador estaría formado por un "ángulo muy marcado que avanza sobre el vacío" en la habitación 15 de Rincón de Vallejo. La pared del "mirador" tendría "un alto considerable", y visto desde abajo aparentaría una torre. Sin embargo, este sector de la construcción no presenta características arquitectónicas que permitan su identificación como tal (ver Michieli et al. 2005, fotografías 55 y 56). A ello se agrega que, de haber existido, habría sido un elemento meramente decorativo debido a que la topografía de la cuesta impide (con mirador o sin él) el avistamiento de los sectores adyacentes a la Quebrada de Conconta.

Igualmente, difícil de sostener es la propuesta referida al depósito para crianza de cuyes o para acciones rituales. Dado que simplemente se trata de un espacio "en forma de cubo (sic) de 1,20 x 0,90 x 0,70 m con una abertura trapezoidal en la base y otra en la parte superior" (Michieli et al. 2005:29) en el que no se hallaron excrementos o huesos de cuyes, restos de combustión o elementos rituales, la interpretación propuesta carece de fundamento.

\section{Ubicación de las estructuras principales}

Las estructuras más importantes son las que corresponden al sitio Rincón de Vallejos (o A35), que se localiza en la parte central de la empinada cuesta y presenta alrededor de 20 habitaciones (Figura 5). El sitio se encuentra absolutamente expuesto a las inclemencias climáticas, en un sector totalmente inadecuado para el traspaso de la cuesta a pie o con animales. En efecto, la cuesta tiene un declive de aproximadamente $40^{\circ}$ y una longitud de aproximadamente 1500 metros (la del camino actual que la atraviesa es de unos $7 \mathrm{~km}$ ), y su subida habría insumido un esfuerzo extremadamente grande. Una vez en la cima, el trayecto hacia el Valle del Cura requería sortear un 
terreno muy irregular y de difícil tránsito. En cambio, siguiendo el fondo de la quebrada se podía subir con mayor comodidad por su extremo proximal y luego cruzar en línea recta hacia el Valle del Cura, con un importante ahorro de kilómetros y esfuerzo.

Por otra parte, una vez alcanzado el techo de la cuesta habrían restado por lo menos entre 40 y $50 \mathrm{~km}$ para llegar al actual límite internacional en el sector del Paso de las Tórtolas (siguiendo el camino propuesto por las autoras) y algunos más para acceder a algún sitio apropiado para el descanso en la vertiente oriental. Aun cuando se partiera desde las estructuras ubicadas en la base de la cuesta (A27) ese trayecto resultaba impracticable en el término de un día, por lo que era indispensable contar con un sitio intermedio, utilizado como última posta antes de cruzar la cordillera. A pesar de su búsqueda (Michieli 2015) ese sitio no ha sido encontrado en el sector correspondiente, por lo que cabría dudar seriamente de su existencia.
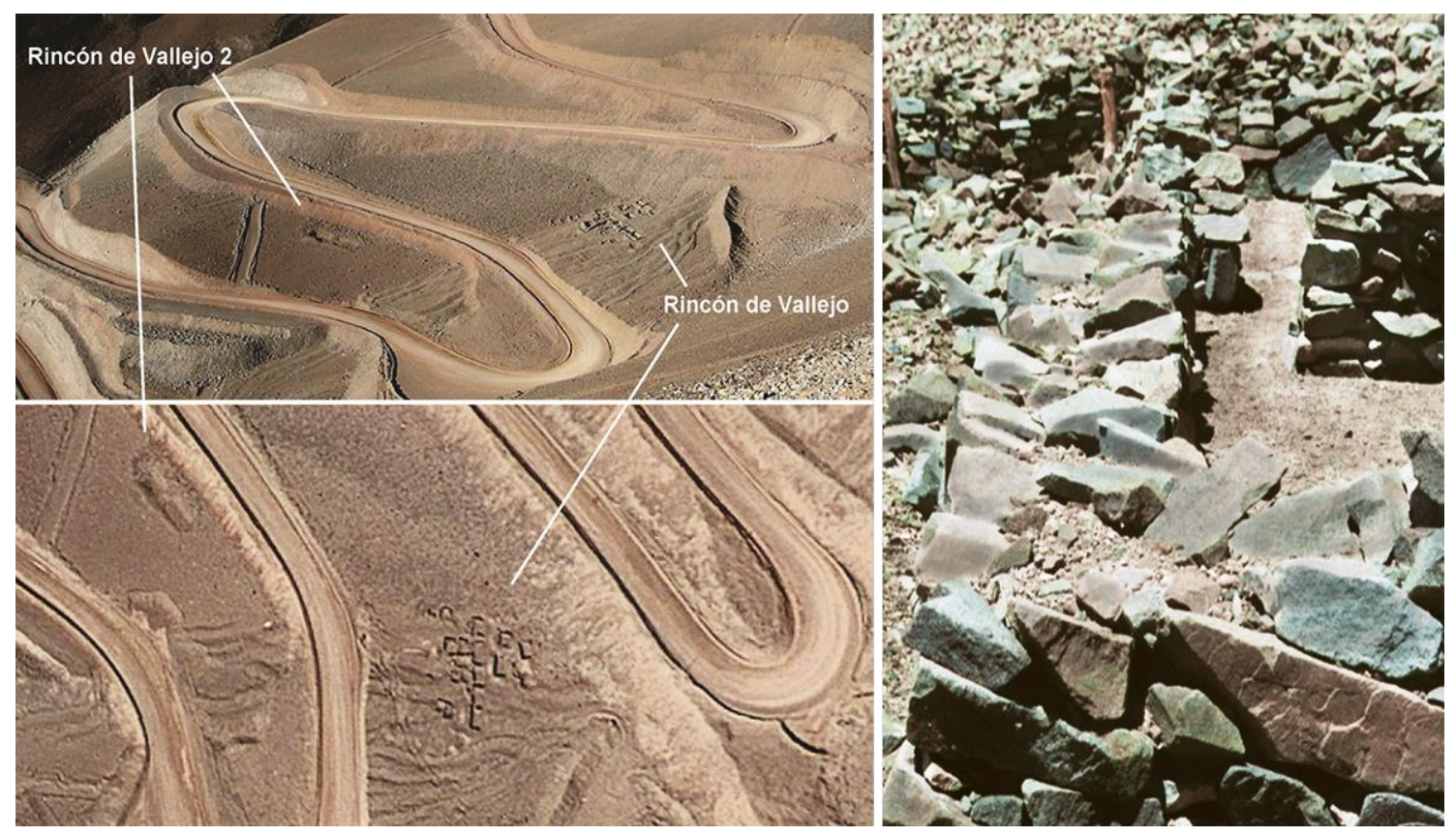

Figura 5. A la izquierda, vistas de los sitios Rincón de Vallejo y Rincón de Vallejo 2. A la derecha, vista parcial de un sector de Rincón de Vallejo (modificada de Revista La Universidad 13, 2013, Universidad Nacional de San Juan).

Por lo tanto, dado que uno de los tramos de camino incaico postulados se habría ubicado en las Vegas de Aguilar (entre el sitio Quebrada de las Máquinas y la cuesta de Vallejo), cabría especular que, de haber sido planificada, la construcción de aquel sitio intermedio no comenzó de manera simultánea con la de las restantes estructuras, incluido el camino que llevaría a ella. Sería, por lo tanto, un llamativo caso de construcción de un camino que llevaba hacia un sitio que aún no se había comenzado a levantar. 
En definitiva, resultan muy extraños el lugar elegido para acceder al sector alto y la secuencia de construcción de las obras. En realidad, como se verá más adelante, la ubicación de las habitaciones de Rincón de Vallejos es muy difícil de comprender como parte de un patrón de asentamiento prehispánico.

\section{Contextos arqueológicos de los sitios}

La inspección ocular de la superficie interna y externa de las estructuras de Rincón de Vallejo, Cuesta de Vallejo, A19/17 y A13 fue exhaustiva, a pesar de lo cual no se habría registrado material arqueológico "fuera de las ruinas mismas" (Michieli et al. 2005:18). Sin embargo, el material arqueológico recuperado fue diverso y abundante, aunque exclusivamente correspondiente a momentos históricos: palos y postes de rollizo, vainas servidas de proyectiles de armas de fuego de diferente calibre, huesos de guanaco y de animales domésticos cortados con sierra, abundante cantidad de restos de calzados (especialmente alpargatas) viejos y reparados con cubiertas de automóvil, recortes de estas cubiertas, leña, latas de conservas, recipientes de vidrio y plástico, tapas de botella, restos de antiparras, fragmentos de lona y otras telas, alambres y clavos, trozos de chapa, carozos de durazno, pilas, un tenedor, un detonador y un recipiente con pastillas de color blanco y "basura de época muy reciente" (Michieli et al. 2005: 18).

De manera similar, en las excavaciones de Rincón de Vallejo se registró un nivel superficial de hasta $10 \mathrm{~cm}$ de profundidad que sólo brindó "basura moderna", restos de tablas con clavo, restos de leña, huesos de vacuno cortados con sierra, papeles metalizados -asociados a envolturas de chocolate y paquetes de cigarrillos- y clavos (Michieli et al. 2005:19). Por debajo de ese nivel no se halló sedimentación en el interior de las habitaciones, lo que sugiere que el único componente ocupacional de estos sitios es el asociado con el registro antes descripto. En ningún caso (ni en las áreas correspondientes a las construcciones ni en las aledañas a los tramos de camino) fue posible localizar materiales de factura indígena (ya se trate de restos incaicos o de materiales vinculables con las etnias locales).

Esta total ausencia de registro arqueológico incaico asociado a sitios asignados a ese período fue oportunamente explicada por la conjunción de varias ideas: a) en general dentro de los sitios incaicos se encontrarían pocos restos arqueológicos, lo cual estaría vinculado "obviamente con el corto desarrollo temporal que tuvo el imperio incaico". La escasez o ausencia sería más frecuente en sitios de altura sometidos a problemas de erosión. b) Estas construcciones son más modernas que los otros sitios incaicos de la región (como los de San Guillermo, Tocota, y los valles longitudinales precordilleranos de San Juan), en los que sí hubo tiempo para que se depositaran restos arqueológicos incaicos. c) Los sitios de Conconta se habrían levantado muy cerca de la caída del imperio incaico, hacia 1532, y habrían sido abandonados súbitamente al llegar la noticia de la conquista española del Cuzco (Michieli et al. 2005: 26-28). 
Esta posición es muy difícil de sostener. En principio, no existen otros sitios incas en la región que no contengan registro arqueológico vinculado con el dominio incaico (ni siquiera los ubicados en los sectores más altos, como la Reserva San Guillermo). Tampoco conocemos en el área andina algún ejemplo de un sitio incaico que carezca absolutamente de registro arqueológico indígena asociado. La inexistencia absoluta de material indígena no puede explicarse si se considera que los sitios son incaicos: por más súbita y planificada que haya sido la construcción de las habitaciones, es imposible que, de haber sido realizadas por indígenas, no quedaran manifestaciones culturales de factura prehispánica. Aun cuando se ejecutara con suma rapidez, la construcción de estos sitios habría requerido varias semanas de trabajo; mientras tanto, los constructores indígenas habrían tenido que desarrollar actividades vinculadas con su propia manutención (talla y uso de instrumentos, cacería, reiterados episodios de alimentación, etc.) cuyo resultado inevitable debió ser el abandono en la zona (o dentro de los sitios) de claras evidencias de su presencia. Sin embargo, no sólo no se hallaron elementos incaicos, sino que tampoco aparecieron materiales indígenas de ningún tipo dentro o en torno a las decenas de recintos relevados.

Por el contrario, todo el registro cultural obtenido es de época muy reciente y se encuentra en el único componente de ocupación del sitio. En definitiva, la propia información aportada por las autoras indica claramente que el origen de estas estructuras no puede vincularse con la ocupación indígena prehispánica local.

Finalmente, la asociación de la reutilización con la identificación de los sitios como incaicos presenta claramente un problema de lógica, y cada caso debe ser estrictamente evaluado en función de las evidencias disponibles.

\section{Características de la vialidad anexa}

Los tramos principales de "camino incaico" identificados en la zona son tres. Se trata de segmentos de características y longitud variables (Michieli et al. 2005: 15, 1718), relativamente cercanos a las estructuras mencionadas. Uno de estos tramos (A15A14) tiene una longitud de 48,70 m y presenta "muro de sostenimiento de terraplén con pirca de piedra de dos hileras sobre el lado que da al arroyo". El tramo de A11-A5 recorre $114 \mathrm{~m}$ sobre la margen izquierda del Arroyo Conconta, con un ancho promedio de 2,30 m. Presenta "muro de sostenimiento conformado por una pirca que por lo general es de una sola hilera", de hasta $0,40 \mathrm{~m}$ de alto. En su extremo más bajo este tramo es simplemente una huella despedrada de 2,5 $\mathrm{m}$ de ancho. El tercer segmento se encuentra sobre los 4100 m s.n.m., entre el Portezuelo de Concota y el Valle del Cura, unos $500 \mathrm{~m}$ al este de la Vega de Aguilar. Tiene $110 \mathrm{~m}$ de largo y 2,30 m de ancho y está "delineado por ambos costados con piedras de mediano tamaño". Llamativamente, si bien este segmento está alejado de las construcciones de la quebrada, al igual que los anteriores, se encuentra a la vera del camino actual. 
En todos los casos, la base de la adscripción étnica parece ser la cercanía espacial con las construcciones analizadas. En contraposición a la factura incaica propuesta, si se tienen en cuenta algunos aspectos puede argumentarse que, por el contrario, estos fragmentos de camino son enteramente modernos:

a) La forma más segura de determinar la presencia de vialidad incaica es identificar los sitios incaicos asociados o hallar restos materiales incaicos indudable y reiteradamente asociados a los caminos (García 2011, 2017; González 2017; Vitry 2004). En el presente caso ninguno de los requisitos se cumple, lo que sugiere un origen distinto al propuesto para estos segmentos viales.

b) La gran inversión de trabajo sugerida para la construcción de algunos tramos difiere significativamente de la observada en la vialidad incaica de la región, en la que sólo se constata la limpieza de un sector de ancho variable entre 1 y $2 \mathrm{~m}$. Esta situación se registra en todos los segmentos conocidos en la región (por ejemplo, el tramo ubicado entre los tambos de Ranchillos y Tambillos, en el Valle de Uspallata, la senda que se dirige hacia el sur del tambo de Tocota y que fragmentariamente puede observarse hasta las cercanías del límite entre Mendoza y San Juan, etc.). Por otra parte, esta falta de jerarquización de tramos viales mediante la incorporación de atributos especiales (elementos demarcatorios, tapizado, etc.) se verifica no sólo en tramos asociados a sitios estratégicos para el control de sectores específicos (e.g. Paso del Lámar, El Acequión) o para la comunicación o explotaciones económicas determinadas (sitios del área de San Guillermo), sino también en aquellos relacionados con lugares que presentan un fuerte componente ideológico (e.g. Cerro Aconcagua, Schobinger 2001; Stehberg y Sotomayor 2002-2005).

c) Otro aspecto que llama la atención es que, como señalan las propias autoras, dado que en el Valle del Cura no se han hallado sitios ni vestigios incaicos, y que la construcción de los sitios de la parte alta de la Quebrada de Conconta no fue finalizada, la elaboración de la red vial zonal habría antecedido a la de los tambos. O sea que se habría invertido una gran cantidad de tiempo y un esfuerzo considerable para construir la vialidad imperial antes de que los sitios asociados a la misma fueran reutilizados o terminados, estuvieran funcionalmente aptos para ser utilizados, o incluso existieran (ver supra).

d) Las características de los segmentos de camino observados en el área no difieren de las que quedan como evidencia luego del mantenimiento de sendas viales de montaña mediante la utilización de maquinaria moderna. Como es sabido, los caminos localizados en los fondos de quebrada son frecuentemente afectados por las crecidas de los arroyos y muchas veces su arreglo implica cambios en la ubicación de las sendas y el abandono de algunos segmentos inutilizados (por ejemplo, por cortes transversales producidos por el agua). Esto se observa claramente en el caso de A11-A5, donde habría "una rectificación del camino incaico mediante una variante a un nivel superior", lo que permite apreciar dos 
tramos superpuestos sobre la ladera, ambos con muro de sostenimiento. Quebrada abajo, a esta senda "se le ha superpuesto una huella de época más reciente (...) y posteriormente desaparecen confundiéndose con los reiterados trazados de huellas y caminos diversos". De manera similar, la demarcación con rocas de pequeño o mediano tamaño observada en el tramo superior no es diferente de la registrada en casos modernos, ya sea por desplazamientos de material producidos por maquinaria pesada durante tareas de mantenimiento o por señalamientos de valor estético realizados por ocupantes de puestos actuales (por ejemplo, el realizado en el Valle del Cura en las cercanías del puesto de Gendarmería de la localidad de Sepultura).

En suma, las consideraciones anteriores indican que el origen de los supuestos tramos de vialidad incaica de la Quebrada de Conconta no presenta relación con la utilización o explotación indígena de la zona, y que su cronología sería más reciente.

\section{El contexto arqueológico regional}

La articulación con el registro arqueológico regional es un aspecto muy importante para dilucidar este caso. En efecto, los sitios de la parte alta de la Quebrada de Conconta deberían estar integrados a una red mayor, a través de componentes localizados en el resto de la quebrada y en el piedemonte cordillerano. Sin embargo, un análisis del registro arqueológico regional indica una situación diferente. Por una parte, no existen restos incaicos en las zonas aledañas a la Cuesta de Vallejo (Figura 1). Por el norte los restos incaicos más cercanos hasta ahora conocidos se encuentran a más de $60 \mathrm{~km}$, en la zona de San Guillermo; hacia el oeste, a unos $25 \mathrm{~km}$ en línea recta se encuentra el Cerro Tórtolas, en cuya vertiente occidental se hallaron evidencias de un sacrificio de altura; por el este no se han hallado restos incaicos en toda la Quebrada de Conconta, ni en el piedemonte (el sitio inca estudiado más cercano -Paso del Lámar- se ubica a unos $125 \mathrm{~km}$ de distancia), en tanto que por el sureste el sitio más cercano es Tocota, a aproximadamente $75 \mathrm{~km}$ en línea recta.

Restos indígenas correspondientes a grupos locales de distintos momentos previos a la dominación inca (construcciones, restos líticos y cerámicos, arte rupestre, etc.) se han registrado sólo en la parte distal de la Quebrada de Conconta (donde ésta sale al piedemonte). Esto indicaría el escaso interés que despertaba la ocupación o explotación de los tramos medio y superior, y la posible desestimación de esta vía para acceder a los sectores altos cordilleranos. Adicionalmente, el análisis del sitio Quebrada de las Máquinas (Michieli 2015), situado en las proximidades del Cerro Tórtolas y considerado como evidencia del tránsito cordillerano que conectaba la vertiente occidental con los valles bajos sanjuaninos a través de la Quebrada de Conconta, tampoco ha brindado elementos incaicos. 
Por el contrario, a lo largo de toda la quebrada se observan construcciones modernas. La falta de explotación minera en la quebrada permite desvincular de esta actividad a tales estructuras, las que, consecuentemente, deben asociarse a otro tipo de emprendimiento reciente (ver infra).

El panorama anterior muestra claramente que los sitios "incaicos" de Conconta se encuentran particularmente aislados del conjunto de evidencias incaicas de la región, y esta desvinculación constituye otro elemento negativo al momento de contrastar la asignación étnica propuesta.

\section{Información oral reciente}

La búsqueda de datos acerca de las construcciones analizadas permitió el acceso a un informe datado en 2001, en el que se volcaron importantes datos provenientes de algunas de las personas que trabajaron en la apertura del camino que recorre longitudinalmente la Quebrada de Conconta. Según este informe, preparado por el señor Tráncito Herrera, empleado de la firma Homestake (vinculada con servicios mineros), "este camino se comenzó a construir en septiembre de 1955". El informe indica además que: "todas las pircas que están a lo largo de dicho camino desde Tudcum y hasta Los Champones han sido hechas por los obreros de la Compañía Minera San Juan para refugios, que eran utilizados como cocina. Los más importantes de estos refugios eran los de Peñasquito, Vega de Fauto, Rondadero y el del Desarrollo de Vallejo (...). Las primeras pircas de este Desarrollo se comenzaron a hacer a fines de la temporada del año 1956" (Herrera 2001). Cabe señalar que Los Champones se sitúa en el Valle del Cura, luego de traspasar la Cuesta de Vallejo. Muy interesantes son algunas apreciaciones vinculadas con las condiciones ambientales del sector. Así, según el informe, "al año siguiente, al regresar al lugar, se encontraron que estaba todo congelado, por lo que tuvieron que romper la puerta. Las herramientas estaban cubiertas de hielo, lo que les dificultó su recuperación" (Herrera 2001).

Este informe deja claro que el camino localizado en el fondo de la Quebrada de Tocota que permitía sortear la marcada subida de la Cuesta de Vallejo y acceder al Valle del Cura, data de mediados de la década de 1950, y que las pircas modernas que se observan a lo largo de la quebrada son de esa época y están vinculadas con la construcción del camino.

En concordancia con lo expresado por este informe, no existe mención alguna en la documentación histórica (donde generalmente los sitios indígenas aparecen mencionados como "casas", "tambos" o "tamberías") o en la bibliografía regional, ni se encuentran señalados en la cartografía local, lo que contrasta notablemente con la importancia que habrían tenido por su localización y función. 


\section{Consideraciones finales}

Desde un punto de vista arqueológico, toda la información disponible indica claramente que las construcciones del tramo superior de la Quebrada de Conconta no son de origen incaico: no hay otros sitios incas en las zonas adyacentes, no hay restos incaicos vinculados con las construcciones o los tramos de camino (ni en las zonas adyacentes), ni hay documentos (a diferencia de otras áreas; García 2005) que indiquen la presencia de un sitio incaico en ese lugar. Por más rápida que haya sido la ejecución de estos sitios, su construcción habría requerido varias semanas de trabajo, que habrían dejado algún registro de las actividades directa o indirectamente vinculadas con la obra. Por el contrario, no sólo no se hallaron elementos incaicos sino que tampoco aparecieron materiales indígenas de ningún tipo dentro o en torno a las decenas de recintos relevados. Tampoco resulta comprensible la distribución de varios sitios "incaicos" en unos pocos kilómetros, y su total ausencia en los territorios adyacentes, como si el conjunto pudiera de alguna manera desvincularse de los espacios aledaños. En realidad, la información arqueológica regional conocida sugiere que posiblemente la Cuesta de Vallejo ni siquiera era una vía de comunicación utilizada por los indígenas para acceder al Valle del Cura.

El registro arqueológico de la Quebrada de Conconta es totalmente coherente con la información oral reciente disponible, y ambas vías de evidencias señalan claramente que la construcción de estos recintos se realizó a mediados del siglo XX, en relación con la del camino destinado a atravesar la Cuesta de Vallejo y acceder al Valle del Cura. De esta manera se explican tanto la presencia de construcciones recientes a lo largo de toda la quebrada como el hecho de que en los sectores medio y alto de la quebrada estos sitios contengan exclusivamente material arqueológico moderno. En este contexto, los tramos de camino amurados no son más que partes de ese camino, construidas de manera tal de asegurar su durabilidad en las márgenes del Arroyo Conconta, y las construcciones (tal como indica el informe) serían lugares de refugio relacionados con el avance de la construcción del camino.

Con respecto a las pautas de identificación del registro arqueológico del período incaico, el caso analizado evidencia la necesidad de contar con elementos cuyo origen esté indudablemente ligado al control incaico (e.g., cerámica de determinados estilos probadamente adscribibles a tal período, contextos locales y regionales que permitan argumentar razonablemente a favor de una relación con la dominación incaica, fechados radiocarbónicos, etc.) y de considerar con suma cautela elementos aislados (en este caso, arquitectónicos) cuya asignación cultural sea susceptible de múltiples interpretaciones.

Agradecimientos: Este trabajo se enmarca en proyectos de investigación financiados por CICITCA-UNSJ, SECTYP-UNCuyo y CONICET (PUE 2292016010 Recursos de la provincia de San Juan: conflictos de uso, peligros y prioridades de conservación, su estudio a dos escalas). Agradezco especialmente las valiosas sugerencias de los evaluadores. 


\section{Bibliografía citada}

Bárcena, J.R.

1979 Informe sobre recientes investigaciones arqueológicas en el N.O. de la Provincia de Mendoza (Valle de Uspallata y zonas vecinas) (con especial referencia al periodo incaico). Actas del VII Congreso de Arqueología de Chile, T. II, pp. 661-692. Kultrún, Santiago de Chile.

1988 Investigación de la dominación incaica en Mendoza. El tambo de Tambillos, la vialidad anexa y los altos cerros cercanos. Espacio, tiempo, forma I: 397-426.

1998 El tambo Real de Ranchillos, Mendoza, Argentina. Xama 6:1-52

2001 Consideraciones generales y avances particulares sobre la dominación incaica en el centro oeste argentino. Actas XIII Congreso Nacional de Arqueología Argentina, vol. 1, pp. 277-296. Córdoba.

2005 Avances 2002-2003 sobre el conocimiento arqueológico y etnohistórico de la dominación inka en el Centro Oeste Argentino, extremo austral oriental del Tawantinsuyu. Xama 15-18: 119-149

2006 Arqueología de la arquitectura inka del Centro Oeste Argentino. Actas del XVIII Congreso Nacional de Arqueología Chilena, vol. 2, pp. 877-886. Valdivia.

2008 Arqueología del sitio inka de La Alcaparrosa, Parque Nacional San Guillermo. Provincia de San Juan, República Argentina. Incihusa (Conicet), Mendoza.

Berberián, E., J. Martín de Zurita y J. Gambetta

1981 Investigaciones arqueológicas en el yacimiento incaico de Tocota (Prov. de San Juan, Rep. Argentina). Anales de Arqueología y Etnología XXXII-XXXIII: 173-210.

Cahiza, P. y M.J. Ots

2005 La presencia inka en el extremo sur oriental del Kollasuyo. Investigaciones en las tierras bajas de San Juan y Mendoza, y el Valle de Uco -Rca. Argentina. Xama 15-18: 217-228.

Debenedetti, S.

1917 Investigaciones arqueológicas en los valles preandinos de la provincia de San Juan. Publicaciones de la Sección Antropología 15. Facultad de Filosofía y Letras, Universidad de Buenos Aires.

Durán, V., P. Novellino, A. Gil, L. Menéndez, V. Bernal e I. Pérez

2014 Estudios arqueológicos y bioarqueológicos en el valle del río Gualcamayo en el norte de San Juan, Argentina. Arqueología de Ambientes de Altura de Mendoza y San Juan (Argentina), V. Cortegoso, V. Durán y A. Gasco (coord.), pp. 361-399. Ediunc, Mendoza.

Gambier, M. y C. Michieli

1986 Construcciones incaicas y vicuñas en San Guillermo. Un modelo de explotación económica de una región inhóspita. Publicaciones 15: 3-78.

1992 Formas de dominación incaica en la provincia de San Juan. Publicaciones 19: 11-19. 
García, A.

2005 Hallazgo del "Fuerte del Inga" del Acequión. Actas del VII Encuentro de Historia Argentina y Regional, pp. 150-159. Facultad de Filosofía y Letras, UNCuyo, Mendoza.

2007a ¿Ocupación incaica en la Quebrada de Conconta? Una propuesta alternativa. XVI Congreso Nacional de Arqueología Argentina III: 521-527. Jujuy.

2007b El control incaico del área del Acequión (sur de San Juan). XVI Congreso Nacional de Arqueología Argentina, tomo II, pp. 487-491. Jujuy.

2011 El camino del inca entre Tocota y Villa Nueva (Valle de Iglesia, San Juan). Revista del Museo de Antropología 4: 89-98.

2015 Estudios arqueológicos en la frontera sudoriental del Tawantinsuyu: la excavación de Pedernal-Sitio 2. Revista Española de Antropología Americana 45 (2): 439455.

2017 La vialidad incaica de la provincia de San Juan (Argentina). Boletín del Museo Chileno de Arte Precolombino 22 (1):137-150. http://boletinmuseoprecolombino.cl/ wp/wp-content/uploads/2017/08/07Garcia.pdf

González Godoy, Carlos.

2017 Arqueología vial del Qhapaq Nan en Sudamérica: Análisis teórico, conceptos y definiciones. Boletín del Museo Chileno de Arte Precolombino 22 (1): 15-34. http:// boletinmuseoprecolombino.cl/wp/wp-content/uploads/2017/08/01Gonzalez.pdf

Herrera, $\mathrm{T}$.

2001 Primera apertura Camino Tudcum - Valle del Cura. Informe presentado a Homestake. Copia proporcionada por el autor.

Michieli, C.T.

2001 Tambos incaicos del centro de San Juan: su articulación regional. Actas del XIII Congreso Nacional de Arqueología Argentina I, pp. 361-376. Córdoba.

2011 Estudios recientes sobre la conquista incaica en la alta cordillera de San Juan. Arqueología y etnohistoria del Centro-Oeste argentino. Publicación de las VIII Jornadas de Investigadores en Arqueología y Etnohistoria del Centro-Oeste del País, C. Mayol Laferrére, F. Rivero y J. Díaz (comps.), pp. 41-52. Universidad Nacional de Río Cuarto, Río Cuarto.

2015 Articulación del espacio cordillerano: el sitio Quebrada de las MáquinasConfluencia (San Juan, Argentina). Arqueología y Etnohistoria del Centro Oeste Argentino (Coord. por A.M. Rocchietti), pp. 12-21. UniRío, Río Cuarto.

Michieli, C.T., A. Varela y M. G. Riveros

2005 Investigaciones arqueológicas y protección de las instalaciones incaicas de la Quebrada de Conconta (San Juan, Argentina). Publicaciones 27: 3-42.

2010 Investigaciones arqueológicas y protección de las instalaciones incaicas de la Quebrada de Conconta (San Juan, Argentina). Problemáticas de la Arqueología Contemporánea, tomo III (Comp. por A. Austral y M. Tamagnini), pp. 909-911. Río Cuarto. 
Rodriguez, A. y A. García

2016 El registro cerámico del sitio La Invernada: caracterización general e implicancias. Comechingonia 19 (1): 111-130. https://revistas.unc.edu.ar/index. $\mathrm{php} /$ comechingonia/article/view/27373

Schobinger, J. (comp.)

2001 El santuario incaico del Cerro Aconcagua. EDIUNC, Mendoza.

Schobinger, J. y J.R. Bárcena

1971 El 'tambo' incaico de Tambillitos (Prov. Mendoza, Argentina). Actas del VI Congreso de Arqueología Chilena, pp. 397-403. Santiago de Chile

Stehberg, R.

1995 Instalaciones incaicas en el norte semiárido de Chile. Dibam, Santiago de Chile.

Stehberg, R. y G. Sotomayor

2002-2005 Cultos inkaicos en el Valle de Aconcagua (Chile Central). Xama 15-18: 279-285

UNESCO

2014 Decisions adopted by the World Heritage committee at its 38th Session (Doha, 2014). Doha. http://whc.unesco.org/archive/2014/whc14-38com-16en.pdf

Vitry, C.

2004 Propuesta metodológica para el registro de caminos con componentes inkas. Andes 15. http://www.redalyc.org/articulo.oa? id=12701507 (15 abril 2019).

2007 Caminos rituales y montañas sagradas. Estudio de la vialidad inka en el nevado de Chañi, Argentina. Boletín del Museo Chileno de Arte Precolombino 12 (2): 69-84. http://boletinmuseoprecolombino.cl/wp/wp-content/uploads/2015/12/ bol12-207.pdf 